\title{
Invasive Physiotherapy as a Treatment of Spasticity: A Systematic Review
}

\author{
Alberto Javier-Ormazábal (D)', Montserrat González-Platas $\mathbb{D}^{2}$, Elena González-Sierra ${ }^{3}$, \\ Marta González-Sierra ${ }^{4}$

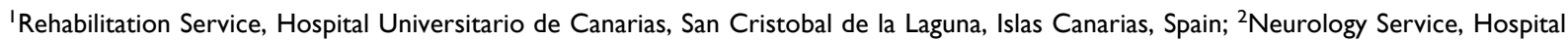 \\ Universitario de Canarias, San Cristóbal de La Laguna, Islas Canarias, Spain; ${ }^{3}$ English Department, University of Valencia, Valencia, Spain; ${ }^{4}$ Home \\ Hospitalization, Service Hospital Universitario de Canarias, San Cristóbal de La Laguna, Islas Canarias, Spain \\ Correspondence: Alberto Javier-Ormazábal, Calle La Rosa no. 4, San Cristóbal de La Laguna, S/C de Tenerife 38203, Spain, \\ Email albert.jaor@gmail.com
}

Introduction: Nowadays, a set of novel physiotherapy techniques have emerged, in which the physical agent used to try to reduce spasticity is applied percutaneously, specifically, through the patient's skin. The aim of this work is to encompass all the invasive techniques used in spasticity in a single article, updating the existing bibliography.

Methodology: A systematic review was carried out between December 2020 and April 2021 in the Web of Science, Scopus and PubMed databases, selecting the clinical trials that used acupuncture, electroacupuncture or dry needling as a treatment for spasticity. Sixteen clinical trials were included, summarizing all the study characteristics and the outcome measures, at last the evidence was described for their results.

Results: Most of the studies find a difference of significant decrease in spasticity between the subjects of the experimental groups. Only four studies found no significant changes in spasticity. All the studies are carried out together with the conventional physiotherapy treatment in spasticity.

Conclusion: Treatment with invasive physiotherapy, combined with conventional physiotherapy, seems to have positive effects in reducing spasticity, although more studies are needed to improve the heterogeneity of the interventions and to assess their long-term effectiveness.

Keywords: acupuncture, electroacupuncture, dry needling, spasticity, invasive physiotherapy

\section{Introduction}

Spasticity is a sensory motor alteration defined as a motor disorder, distinguished by a speed-dependent increase in tonic stretch reflexes, being one of the signs in upper motor neuron syndrome (UNMS). ${ }^{1}$ UNMS can be described as a lesion that occurs in the upper levels of the central nervous system (CNS). The transition from hypotonic and flaccidity to spasticity is slightly controversial, it is thought to be related to the parapyramidal pathways and that the damage will always be present in the CNS, motor cortex, internal capsule, brain stem, or spinal cord. ${ }^{2}$

Clinically, spasticity is considered as a combination of the so-called "positive" phenomena: tonic (hypertonia) and phasic (clonus, spasms), and "negative" phenomena: paralysis and loss of motor control, presented after the injury of the first motor neuron ${ }^{3,4}$ spasticity distinguished by: ${ }^{5}$

- Gradual development in 6-8 weeks in the case of stroke and 2-8 weeks in cranioencephalic trauma (TBI) and gradually in the case of multiple sclerosis.

- Altered motor regulation

- Pain

- Involuntary contractions movements 
We found variations in the symptoms depending on the lesion location, being able to distinguish three different clinical manifestations:

- Lesion of the cortical, internal capsule or torso above the bulbo-protuberancial reticular nucleus: moderate spasticity is observed with a predominance of extensor activity (typical of hemiplegia of vascular etiology).

- Incomplete spinal cord injury: significant spasticity is observed with predominantly extensor motor patterns.

- Complete spinal cord injury: presents a great variety of clinical aspects, due more to the location of the injury than to its etiology. ${ }^{4,6}$

This type of condition can become disabling and affects about $85 \%$ of patients with multiple sclerosis, about $35 \%$ of patients with chronic hemiplegia and between 65 and $78 \%$ of subjects with spinal cord injury. ${ }^{3}$ In addition, it has a dynamic and changing character over time and evolves towards chronicity with the onset of fibrosis with retractions, joint deformities and pain. ${ }^{5}$

In 2007, it was estimated that in Spain between 300,000 and 400,000 people had this type of injury, that is, 10 out of every 1000 inhabitants will live with this health problem.

Regarding the treatment of spasticity, it comprises a multidisciplinary approach where we can include: ${ }^{5}$

- Rehabilitation: Improves disability and quality of life. The techniques used in physiotherapy and occupational therapy are essential to reduce spasticity and prevent complications resulted from it.

- Pharmacological treatment: among the oral drugs used are baclofen, tizanidine, clonidine, diazepam, gabapentin and dantrolene sodium, neuromuscular blockade by intramuscular injection of botulinum toxin type A (BoNT-A).

- Surgery: reserved for those cases of severe spasticity.

In recent years the role of physiotherapy has diversified and expanded considerably, always being at the side of people who suffer from different situations of disability. Physiotherapists are in a continuous search, through research, to expand and refine their knowledge to offer better therapeutic options to help. ${ }^{7,8}$

Currently, a set of physiotherapy techniques have emerged, in which the physical agent used to try to reduce spasticity is applied percutaneously, specifically, through the patient's skin. This set of techniques is included within the expression coined by Mayoral-del-Moral and Lacomba in 2001, ${ }^{9}$ called "Invasive Physiotherapy", where the physical agent used, can only be the mechanical stimulus of the needle, or a combination of the stimulus and the application of electrical current that goes through one or more. ${ }^{9}$

Within this set of spasticity treatment techniques, we can highlight:

1. Acupuncture: application of needles on special skin points, called "channels" or "meridians". ${ }^{10}$ Currently there are studies indicating the efficacy of acupuncture as an alternative in the treatment of neurological diseases. ${ }^{11}$ The systematic reviews (SR) and meta-analyses carried out by Lim et $\mathrm{al}^{12}$ and Shi et $\mathrm{al}^{13}$ in addition to the SR carried out by Rodríguez-Mansilla et $\mathrm{al}^{11}$ where articles on patients with spasticity after a cerebrovascular accident are detailed, they conclude that acupuncture can be effective to lower muscle tone in spasticity.

2. Electroacupuncture (EA): is a recent variation of acupuncture, which consists of applying pulses of electrical current, with an intensity of $1-2 \mathrm{~mA}$ and a frequency around $5 / 20 \mathrm{~Hz}$, through needles inserted into specific acupuncture points. ${ }^{14,15}$

3. Dry needling $(\mathrm{DN})$ : technique that uses a fine thread like needle to penetrate the skin and stimulate myofascial trigger points, as well as connective tissue, for the management of neuromuscular conditions ${ }^{16}$ and spasticity. ${ }^{17}$ Regarding the recent SRs carried out in this treatment modality, the authors conclude that there is little evidence that DN reduces spasticity, pain and range of motion, both in the upper and lower limbs, in adults who have suffered a stroke and present this. ${ }^{18,19}$ 
This work aims to deepen the available evidence regarding the rehabilitative treatment of spasticity using physiotherapy techniques characterized as "invasive physiotherapy". In addition, it is intended to unify those physiotherapy techniques that use a needle as a spasticity treatment in a single publication, owing to the fact that up to now there are no scientific articles in which these techniques are unified in the treatment of this pathology.

\section{Methodology \\ Design}

This systematic review has been carried out following the PRISMA recommendations. ${ }^{20}$ The bibliographic reference research of the available scientific literature was carried out between December 2020 and April 2021.

\section{Information Sources and Research Strategy}

The search was carried out in the following databases and platforms: Web of Science, PubMed and Scopus.

The descriptors used in the search strategy were:

\section{Web of Science}

"Acupuncture" AND "Spasticity" with seven results, "Electroacupuncture" AND "Spasticity" with three results, "Dry needling" AND "Spasticity" with 11 results. To refine the search, the document type was indicated as "Clinical Trials".

\section{Scopus}

"Acupuncture" AND "Spasticity", with eight results "Electroacupuncture" AND "Spasticity" with two results "Dry needling" AND "Spasticity" with 75 results. The search was refined looking into fields of "Medicine", "Neuroscience" and "Health Professions".

\section{PubMed}

"Acupuncture" AND "Spasticity" with seven results, "Electroacupuncture" AND "Spasticity" with four results, "Dry needling" AND "Spasticity" with four results. To refine the search, the document type was indicated as "Clinical Trials".

\section{Inclusion and Exclusion Criteria}

The inclusion criteria to carry out this systematic review is the following:

1. Clinical trials

2. English and Spanish articles

3. Study patients are human beings, older than 18 years and present spasticity, due to some neurological disease, both innate and acquired

4. Acupuncture, electroacupuncture and dry needling are included as possible treatments.

The exclusion criteria are the following:

1. Observational studies, case control studies, narrative reviews and meta-analysis

2. Duplicate articles

3. Articles written in a language other than English or Spanish

4. Those where other treatments are combined (except conventional rehabilitation treatment).

\section{Methodological Quality}

To assess the methodological quality of each article, it was applied the PEDro scale (Physiotherapy Evidence Database). ${ }^{21}$ It is composed of 11 items that evaluate the internal validity and the presentation of a correct statistical analysis, assigning it a point or none depending on whether the items are present or not respectively in the evaluated study. The maximum score that a study can receive is 10 despite having 11 items, since the first one reports on external 
validity. ${ }^{22}$ Articles with a score equal or greater than five have a high methodological quality (good, excellent); moderate quality if the score is four or five (fair study), and low quality if the score is less than four (poor study). ${ }^{23}$

\section{Results Description and Analysis Description of the Study Selection Process}

The information flow chart (Annex I) describes the schematic form of the search strategy based on preferred communication articles for systematic reviews and meta-analyses (PRISMA). ${ }^{20}$

After conducting search on all platforms, a total of 121 references were obtained, of which, eliminating duplicates, 112 articles resulted. The information flow diagram (Annex I) shows the results obtained for each descriptor in each of the bibliographic sources consulted. When the title and abstract were read to decide which met the selection criteria, 16 articles were obtained, which were read in full text and included in this systematic review. Ninety-six articles were eliminated as they did not pass the inclusion criteria (Chinese language, the study patients did not have spasticity, they were not clinical trials, the participants were children and they used other therapies such as botulinum toxin).

\section{Internal Validity of the Studies}

As shown in Annex II, Table I, the analysis presents two studies of low quality, ${ }^{24,25}$ two of moderate quality, ${ }^{26,27}$ eight of good quality ${ }^{28-35}$ and four of excellent quality. ${ }^{36-39}$

\section{Description of the Study Sample}

In all the screened studies, the population was adult patients, who presented damage after suffering a stroke, with the presence of spasticity. The sample size of the studies varies between 7 and 120 patients, with a total of 597 patients analyzed, as shown in the study description in Annex III, Table II.

\section{Study Intervention Description}

Regarding the type of intervention used in the screened clinical trials, we outline the previously mentioned invasive techniques, which are included within the term "invasive physiotherapy": acupuncture, electroacupuncture and dry needling. Where they are combined with conventional physiotherapy performed in the treatment of neurological patients: mobility exercises, stretching, strength training, balance training, Bobath therapy, etc. In this work there is no single protocol in terms of the number of sessions in which invasive physiotherapy techniques are introduced, as well as their treatment time. There are studies with just one session, ${ }^{28}$ or between one ${ }^{38}$ and three sessions per week. ${ }^{30}$ The duration of the protocol goes from three ${ }^{38}$ to eight weeks. ${ }^{27}$ In just three studies they use the entire sample to carry out the intervention, ${ }^{24,25,36}$ whereas in the others they randomize the sample in a control group and in an experimental group.

Finally, the intensity of the current in the studies corresponding at electroacupunture modality are not homogeneous. We observe how the frequency of the current, depending on the studies, oscillates between $2 \mathrm{~Hz}^{24} 50 \mathrm{~Hz}^{35}$ and fluctuates between 50 and $100 \mathrm{~Hz}^{30}$

\section{Studies Results Description}

The most frequently used clinical instruments for measuring outcomes were the Modified Asword Scale (MAS), the Fugl-Meyer Assessment (FMA) and the Modified Barthel Index Score (MBI). In most of the studies, can be noticed that there were significant decreases in the MAS after the application of the following protocols in the patients under study. ${ }^{24,26,29,32,34-36,39}$ One study also showed the improvement of pain in the spastic patient where it was shown that the pain decreases significantly 24 and 72 hours after applying a conventional physiotherapy session along with dry needling, in a patient with shoulder hemiplegia. ${ }^{27}$ Finally, in four studies, no statistically significant changes were found between the control group and the intervention group, two of the studies matched the acupuncture modality ${ }^{33,36}$ and two studies matched the electroacupuncture modality. ${ }^{30,36}$

The exact data on the sample size, the study intervention and the description of the results are detailed in Annex III, $\underline{\text { Table II. }}$ 


\section{Discussion}

With the increasing use and fast progress in different fields of the multiple invasive techniques of physiotherapy, ${ }^{9}$ the physiotherapists themselves base their invasive interventions on clinical reasoning, always integrating the existing scientific evidence with their clinical experience. ${ }^{8}$ We could determine that, after this systematic review, there is clear scientific evidence that the use of minimal invasive physiotherapy in the treatment of spasticity may be indicated. With the results obtained in the methodological quality of the screened studies we emphasize that, of the sixteen studies, only two presented low methodological quality, two with moderate quality, and 12 with good-excellent quality (score between 8 and 10).

In the results obtained, it can be observed that in nine studies there is a presence of improvement in spasticity after the intervention performed ${ }^{24-29,32,34,37-39}$ versus four studies in which no significant improvements were found. ${ }^{30,31,33,36}$ With these results, it could be argued that there are a greater number of studies that found an improvement in muscle tone, compared to a smaller number in which no change was found. In this sense, we find similarities with other systematic reviews carried out in the different areas in which our work is focused, where it is highlighted that minimal invasive techniques can play an important role, as a help to conventional physiotherapy treatment, decreasing the spastic muscle tone on a large number of occasions. ${ }^{12,13,16}$

However, what remains to be defined is the exact number of intervention sessions and weeks of these techniques, since there is still no valid established protocol. We can observe in our work how the authors use different protocols in their clinical trials, without following any type of established practice. In this regard, we found coincidences with the systematic review carried out by Rodríguez-Mansilla et $\mathrm{al}^{11}$ where, it is outlined that "medium and long term follow-up studies are necessary to determine the extent of efficacy in acupuncture". In our work, we can emphasize that, indeed, no follow-up is carried out six months or one year after the protocol. Therefore, we agree that future studies are needed to evaluate the long term efficacy of this type of procedure.

Finally, we agree with Valencia-Chulián et $\mathrm{al}^{18}$ in the SR carried out in 2020, on dry needling in the treatment of spasticity, in which minimal invasive therapies have a positive, beneficial, and safe effect in patients who have suffered a stroke, however, it must be carried out in numerous studies with a higher methodological level and present a larger sample.

\section{Limitations}

First of all, it is necessary to outline the limited evidence of invasive physiotherapy in the field of neurological physiotherapy. When searching, we came across a remarkable number of articles written in Chinese. For this reason, perhaps, the results of this SR have been altered. It could be that those articles written in Chinese and not introduced in this review had a higher methodological quality, which would give even more determination to this summary and to the application of these techniques in a daily clinical practice.

Second, the variability of the techniques that has been introduced in this work and the variability of the methodology used, does not make clear a homogeneous method of using invasive techniques to treat spasticity, obtaining multiple variables for the application of these techniques by the physiotherapist, but without an effective and efficient method.

\section{Conclusions}

Despite the unification in this work of the invasive techniques used in physiotherapy for the treatment of spasticity, we cannot confirm that these techniques reduce muscle tone in an isolated way, since in the literature reviewed always they are accompanied by a conventional physiotherapy protocol. Conversely, we cannot establish the benefit of these therapies in the long-term, so future clinical trials are needed to study this effect, in addition to studies where there is a homogeneity of protocols, in order to determine the ideal dose to apply it in the habitual clinical practice.

\section{Funding}

No funding was received for this study. 


\section{Disclosure}

Dr Montserrat González-Platas reports personal fees from Sanofi, Biogen, Roche, and Novartis, outside the submitted work. The authors report no other conflicts of interest in this work.

\section{References}

1. Fernández-Tenorio E, Serrano-Muñoz D, Avendaño-Coy J, Gómez-Soriano J. Transcutaneous electrical nerve stimulation for spasticity: a systematic review. Neurologia. 2019;34(7):451-460. doi:10.1016/j.nrl.2016.06.009

2. Bolaños-Jiménez R, Arizmendi-Vargas J, Calderón-álvarez Tostado JL, Carrillo-Ruiz JD, Rivera-Silva G, Jiménez-Ponce F. Spasticity, physiological and pathophysiological concepts applied to clinical practice. Rev Mex Neurocienc. 2011;12(3):453-460. doi:10.33588/rn.7012.2019474

3. Gómez-Soriano J, Cano-de-la-cuerda R, Muñoz-Hellín E, Ortiz-Gutiérrez R, Taylor JS. Assessment and quantification of spasticity: review of clinical, biomechanical and neurophysiological methods. Rev Neurol. 2012;55(4):217-226.

4. Vivancos-Matellano F, Pascual-Pascual SI, Nardi-Vilardaga J, et al. Spasticity treatment guide. Rev Neurol. 2007;45(6):365-375. doi:10.33588/ rn.4506.2007239

5. Ferrer-Pastor M, Iñigo-Huarte V, Juste-Díaz J, Goiri-Noguera D, Sogues-Colom A, Cerezo-Durá M. Systematic review of the treatment of spasticity in acquired adult brain damage. Rehabilitación. 2020;54(1):51-62. doi:10.1016/j.rh.2019.06.006

6. Gómez-Soriano J, Taylor J. Spasticity after spinal cord injury: review of the pathophysiological mechanismis, diagnostic techniques and current physiotherapeutic treatments. Fisioterapia. 2010;32(2):89-98. doi:10.1016/j.ft.2009.09.002

7. Lista-Paz A, González-Doniz L, Souto-Camba S. What is the role of physiotherapy in the global COVID-19 pandemic? Fisioterapia. 2020;42 (4):167-169. doi:10.1016/j.ft.2020.04.002

8. Garrido FV, Muñoz FM. Invasive Physiotherapy. España: Elsevier SL: Barcelona; 2016.

9. Mayoral-del Moral O, Lacomba MT. Invasive physiotherapy and dry needling. Report on the efficacy of dry needling and the treatment of myofascial pain syndrome and use in physiotherapy. Cuest Fisioter. 2009;38(3):206-217.

10. Cobos-Romana R. Acupunture, electroacupunture, moxibustion and related pain techniques. Rev Soc Esp Pain. 2013;20(5):263-277. doi:10.4321/ S1134-80462013000500006

11. Rodríguez-Mansilla J, Espejo-Antúnez L, Bustamante-López AI. Efficacy of acupunture in spasticity in patients who have suffered and stroke: systematic review. Aten Primaria. 2016;48(4):226-234. doi:10.1016/j.aprim.2015.05.004

12. Lim SM, Yoo J, Lee E, et al. Acupuncture for spasticity after stroke: a systematic review and meta-analysis of randomized controlled trials. EvidBased Complement Altern Med. 2015;2015:870398. doi:10.1155/2015/870398

13. Shi L, Guo L, Zhang H, et al. Acupuncture for poststroke spasticity: a protocol of a systematic review and meta-analysis. Medicine. 2019;98(39): e17124. doi:10.1097/MD.0000000000017124

14. Quiroz-González S, Li L, Xavier AR, Estrada IJ. Electroacupunture and neuromodulation in the spinal cord: implications in neuropathic pain. Rev Int Acupunt. 2017;11(3):85-95. doi:10.1016/j.acu.2017.10.001

15. Cai Y, Zhang CS, Liu S, et al. Electroacupuncture for poststroke spasticity: a systematic review and meta-analysis. Arch Phys Med Rehabil. 2017;98 (12):2578-2589. doi:10.1016/j.apmr.2017.03.023

16. Rahou-El-Bachiri Y, Navarro-Santana MJ, Gómez-Chiguano GF, et al. Effects of trigger point dry needling for the management of knee pain syndromes: a systematic review and meta-analysis. J Clin Med. 2020;9(7). doi:10.3390/jcm9072044

17. Gallego PH, Del Moral OM, Carrión SC. Use of DNHSC (dry needling for hypertonia and spasticity) in the treatment of hypernonia, spasticity and other alterations and dysfunctions of movement of central origin. Physiotherapy. 2011;33(5):189-191. doi:10.1016/j.ft.2011.06.011

18. Valencia-Chulián R, Heredia-Rizo AM, Moral-Munoz JA, Lucena-Anton D, Luque-Moreno C. Dry needling for the management of spasticity, pain, and range of movement in adults after stroke: a systematic review. Complement Ther Med. 2020;52:102-115. doi:10.1016/j.ctim.2020.102515

19. Hall ML, Mackie AC, Ribeiro DC. Effects of dry needling trigger point therapy in the shoulder region on patients with upper extremity pain and dysfunction: a systematic review with meta-analysis. Physiotherapy. 2018;104(2):167-177. doi:10.1016/j.physio.2017.08.001

20. Urrutia G, Bonfill X. PRISMA Declaration: a proposal to improve the publication of systematic reviews and meta-analyses. Clinical Medicine. 2010;135(11):507-511. doi:10.1016/j.medcli.2010.01.015

21. Maher CG, Sherrington C, Herbert RD, Moseley AM, Elkins M. Reliability of the pedro scale for rating quality of randomized controlled trials. Phys Ther. 2003;83(8):713-721. doi:10.1093/ptj/83.8.713

22. Cascaes-da-silva F, Valdivia-Arancibia B, Da-rosa-iop R, Barbosa-Gutierres F, Da-silva R. Scales and lists of evaluation of the quality of scientific studies. Rev cuba inf cienc salud. 2013;24(3):295-312.

23. Moseley AM, Herbert RD, Sherrington C, Maher CG. Evidence for physiotherapy practice: a survey of the Physiotherapy Evidence Database (PEDro). Aust J Physiother. 2002;48(1):43-49. doi:10.1016/S0004-9514(14)60281-6

24. Mukherjee M, McPeak LK, Redford JB, Sun C, Liu W. The effect of electro-acupuncture on spasticity of the wrist joint in chronic stroke survivors. Arch Phys Med Rehabil. 2007;88(2):159-166. doi:10.1016/j.apmr.2006.10.034

25. Fakhari Z, Ansari NN, Naghdi S, Mansouri K, Radinmehr H. A single group, pretest-posttest clinical trial for the effects of dry needling on wrist flexors spasticity after stroke. NeuroRehabilitation. 2017;40(3):325-336. doi:10.3233/NRE-161420

26. Liu W, Mukherjee M, Sun C, Liu H, McPeak LK. Electroacupuncture may help motor recovery in chronic stroke survivors: a pilot study. $J$ Rehabil Res Dev. 2008;45(4):587-596. doi:10.1682/JRRD.2007.11.0181

27. Cuenca-Zaldívar JN, Calvo S, Bravo-Esteban E, Oliva Ruiz P, Santi-Cano MJ, Herrero P. Effectiveness of dry needling for upper extremity spasticity, quality of life and function in subacute phase stroke patients. Acupunct Med. 2020;39:1-10.

28. Mendigutía-Gómez A, Quintana-García MT, Martín-Sevilla M, et al. Post-needling soreness and trigEGr point dry needling for hemiplegic shoulder pain following stroke. Acupunct Med. 2020;38(3):150-157. doi:10.1177/0964528419882941

29. Sánchez-Mila Z, Salom-Moreno J, Fernández-de-las-peñas C. Effects of dry needling on poststroke spasticity, motor function and stability limits: a randomised clinical trial. Acupunct Med. 2018;36(6):358-366. doi:10.1136/acupmed-2017-011568

30. Cai Y, Zhang CS, Zhang AL, Da Costa C, Xue CC, Wen Z. Electroacupuncture for poststroke spasticity: results of a pilot pragmatic randomized controlled trial. J Pain Symptom Manaeg. 2021;61(2):305-314. doi:10.1016/j.jpainsymman.2020.07.034 
31. Wayne PM, Krebs DE, Macklin EA, et al. Acupuncture for upper-extremity rehabilitation in chronic stroke: a randomized sham-controlled study. Arch Phys Med Rehabil. 2005;86(12):2248-2255. doi:10.1016/j.apmr.2005.07.287

32. Wang HQ, Hou M, Bao CL, Min L, Li H. Effects of acupuncture treatment on lower limb spasticity in patients following hemorrhagic stroke: a pilot study. Eur Neurol. 2019;81(1-2):5-12. doi:10.1159/000499133

33. Fink M, Rollnik JD, Bijak M, et al. Needle acupuncture in chronic poststroke leg spasticity. Arch Phys Med Rehabil. 2004;85(4):667-672. doi:10.1016/j.apmr.2003.06.012

34. Zhao JG, Cao CH, Liu CZ, et al. Effect of acupuncture treatment on spastic states of stroke patients. J Neurol Sci. 2009;276(1-2):143-147. doi:10.1016/j.jns.2008.09.018.20

35. Wang BH, Lin CL, Li TM, Lin SD, Lin JG, Chou LW. Selection of acupoints for managing upper-extremity spasticity in chronic stroke patients. Clin Interv Aging. 2014;9:147-156. doi:10.2147/CIA.S53814

36. Xiaoxi L, Xuezhu Z, Kun N, et al. Effect of electro-scalp acupuncture on acute ischemic stroke: a randomized, single blind, trial. $J$ Tradit Chinese Med. 2018;38(1):95-100. doi:10.1016/j.jtcm.2018.02.012

37. Salom-Moreno J, Sánchez-Mila Z, Ortega-Santiago R, Palacios-Ceña M, Truyol-Domínguez S, Fernández-de-las-peñas C. ChanEGs in spasticity, widespread pressure pain sensitivity, and baropodometry after the application of dry needling in patients who have had a stroke: a randomized controlled trial. J Manipulative Physiol Ther. 2014;37(8):569-579. doi:10.1016/j.jmpt.2014.06.003

38. Ghannadi S, Shariat A, Ansari NN, et al. The effect of dry needling on lower limb dysfunction in poststroke survivors. J Stroke Cerebrovasc Dis. 2020;29(6):104814. doi:10.1016/j.jstrokecerebrovasdis.2020.104814

39. Mendigutia-Gómez A, Martín-Hernández C, Salom-Moreno J, Fernández-de-las-peñas C. Effect of dry needling on spasticity, shoulder range of motion, and pressure pain sensitivity in patients with stroke: a crossover study. J Manipulative Physiol Ther. 2016;39(5):348-358. doi:10.1016/j. jmpt.2016.04.006

Degenerative Neurological and Neuromuscular Disease

Dovepress

\section{Publish your work in this journal}

Degenerative Neurological and Neuromuscular Disease is an international, peer-reviewed, open access journal focusing on research into degenerative neurological and neuromuscular disease, identification of therapeutic targets and the optimal use of preventative and integrated treatment interventions to achieve improved outcomes, enhanced survival and quality of life for the patient. The manuscript management system is completely online and includes a very quick and fair peer-review system. Visit http://www.dovepress.com/testimonials.php to read real quotes from published authors.

Submit your manuscript here: http://www.dovepress.com/degenerative-neurological-and-neuromuscular-disease-journal 